Supporting Information

\title{
The Morphology of Thin Films of Aromatic Ellagic Acid and its Hydrogen-Bonding Interactions
}

Eva Bittrich $^{1 *}$, Jari Domke ${ }^{2}$, Dieter Jehnichen ${ }^{1}$, Lars Bittrich ${ }^{1}$, Mikhail Malanin ${ }^{1}$, Andreas

Janke $^{1}$, Petra Uhlmann ${ }^{1,3}$, Klaus-Jochen Eichhorn ${ }^{1}$, Alexis Papamichail ${ }^{4,5}$, Vallery

Stanishev $^{4}$, Vanya Darakchieva ${ }^{4,5}$, Mahmoud Al-Hussein ${ }^{6}$, Marieta Levichkova ${ }^{7}$, Torsten

Fritz $^{2}$, Karsten Walzer

1. Leibniz-Institut für Polymerforschung Dresden e.V., Hohe Str. 10, 01069 Dresden, Germany

2. Friedrich-Schiller-Universität Jena, Institute of Solid State Physics, Helmholtzweg 5, 07743 Jena, Germany

3. Department of Chemistry, Hamilton Hall, University of Nebraska-Lincoln, 639 North 12th Street, Lincoln, NE 68588, USA

4. Terahertz Materials Analysis Center (THeMAC), Department of Physics, Chemistry and Biology, IFM, Linköping University, Linköping S-58183, Sweden

5. Centre for III-nitride technology (C3NiT), Department of Physics, Chemistry and Biology, IFM, Linköping University, Linköping S-58183, Sweden

6. Physics Department and Hamdi Mango Center for Scientific Research, University of Jordan, Amman 11942, Jordan

7. Heliatek GmbH, Treidlerstr. 3, 01139 Dresden, Germany

*Corresponding author: bittrich-eva@ipfdd.de 


\section{Table of Contents}

Supplementary Figures S1 to S15

Corrections of 2D-GIWAXS data as obtained by an area detector __ S3

Simulated powder diffraction pattern of ellagic acid _

2D-GIWAXS of thin films of C60 $(10 \mathrm{~nm})$ and BPAPF $(10 \mathrm{~nm}) \ldots$ S5

Chemical structure of BPAPF — S5

Azimuthal intensity distribution in 2D-GIWAXS measurements __ S6

LEED patterns of a closed monolayer of $\mathrm{C}_{60}$ and additional ellagic acid _ $\quad \mathrm{S} 7$

Supplementary AFM images and analysis —S8

UV-VIS spectrum and optical dispersions of ellagic acid thin films _ S10

Optical modeling of substrates and roughness _ S11

Supplemental IR-Analysis of ellagic acid thin films on Au/glass and on silicon ___ S12

Microscopic images of micrometer-sized ellagic acid crystals _ S13 


\section{Corrections of 2D-GIWAXS data as obtained by an area detector}



Figure S1: Raw detector image of 2D-GIWAXS on $75 \mathrm{~nm}$ ellagic acid on an Au/glass substrate.

Based on the coordinate system presented in "A. Hexemer and P. Müller-Buschbaum, IUCrJ (2015), 2, 106", the correction of the data obtained by an area detector was performed as follows.

Given values:

- Angle of incidence: $\alpha_{i}=0.2^{\circ}$

- Distance sample - detector: $L=110.0 \mathrm{~mm}$

- Wavelength: $\lambda=1.5418 \AA$

- $\quad X, Y$ : coordinates of the detector image

Angle $\psi$ between incident beam and $\boldsymbol{k}_{\boldsymbol{I I}}$ (with $\boldsymbol{k}_{\boldsymbol{I I}}$ the k-vector in the xy-plane of the sample):

$$
\psi=\tan ^{-1} \frac{X}{L}
$$


Angle $\alpha_{f}$ between $\boldsymbol{k}_{\boldsymbol{I I}}$ and $\boldsymbol{k}_{\boldsymbol{f}}$ :

$$
\alpha_{f}=\tan ^{-1} \frac{Y}{\sqrt{X^{2}+L^{2}}}
$$

Components of the scattering vector $\boldsymbol{q}=\boldsymbol{k}-\boldsymbol{k}_{\boldsymbol{0}}$

$$
\begin{gathered}
q_{x}=\frac{2 \pi}{\lambda}\left(\cos \alpha_{f} \cos \psi-\cos \alpha_{i}\right) \\
q_{y}=\frac{2 \pi}{\lambda}\left(\cos \alpha_{f} \sin \psi\right) \\
q_{z}=\frac{2 \pi}{\lambda}\left(\sin \alpha_{f}+\sin a_{i}\right)
\end{gathered}
$$

Conversion of $\boldsymbol{q}_{x}$ and $\boldsymbol{q}_{\boldsymbol{y}}$ to $\boldsymbol{q}_{x y}$ :

$$
q_{x y}=\sqrt{q_{x}^{2}+q_{y}^{2}}
$$

\section{Simulated powder diffraction pattern of ellagic acid}



Figure S2: Simulated powder diffraction pattern of ellagic acid using the crystallographic data of database entry 1149400 of the Cambridge Crystallographic Data Centre (CCDC, UK). The pattern is simulated in Mercury 4.1.0 (CCDC). The FWHM of all reflections were set to $0.27^{\circ}$. This is the experimentally determined FWHM for the (102) reflection of 75 nm EA on gold in Fig. 2 in the original paper, corrected with the instrumental broadening $\left(0.1^{\circ}\right)$ of $\theta-\theta$ measurements at XRD 3003 at IPF Dresden. 


\section{D-GIWAXS of thin films of C60 (10 $\mathrm{nm})$ and BPAPF $(10 \mathrm{~nm})$}
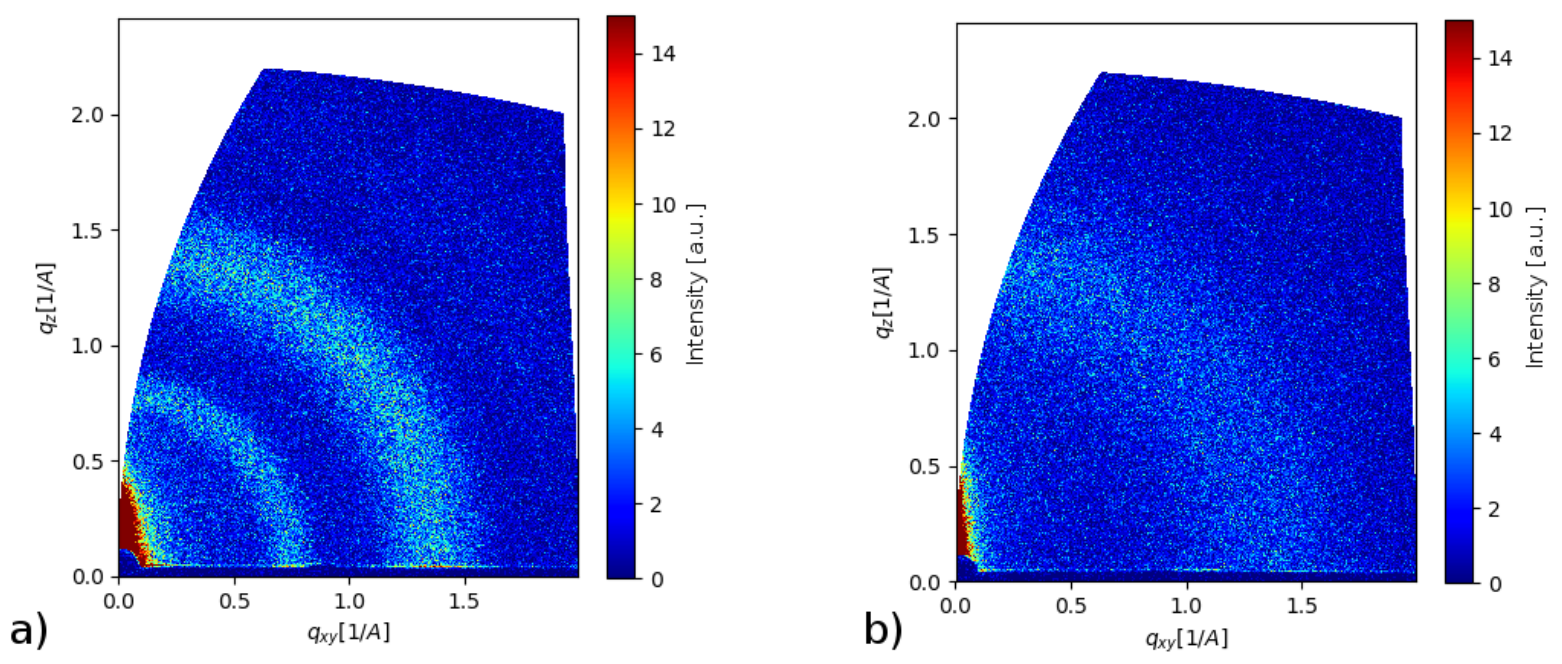

Figure S3: 2D-GIWAXS reference data of $10 \mathrm{~nm} \mathrm{C} 60$ (a) and $10 \mathrm{~nm}$ BPAPF (b) on a silicon substrate. The data are corrected for image distortions by use of an area detector. Thicknesses are nominal values from QCM-measurements in the preparation process. Isotropic Debye Scherrer rings are observed for the $\mathrm{C}_{60}$ film (a), while BPAPF is amorphous (b).

\section{Chemical structure of BPAPF}

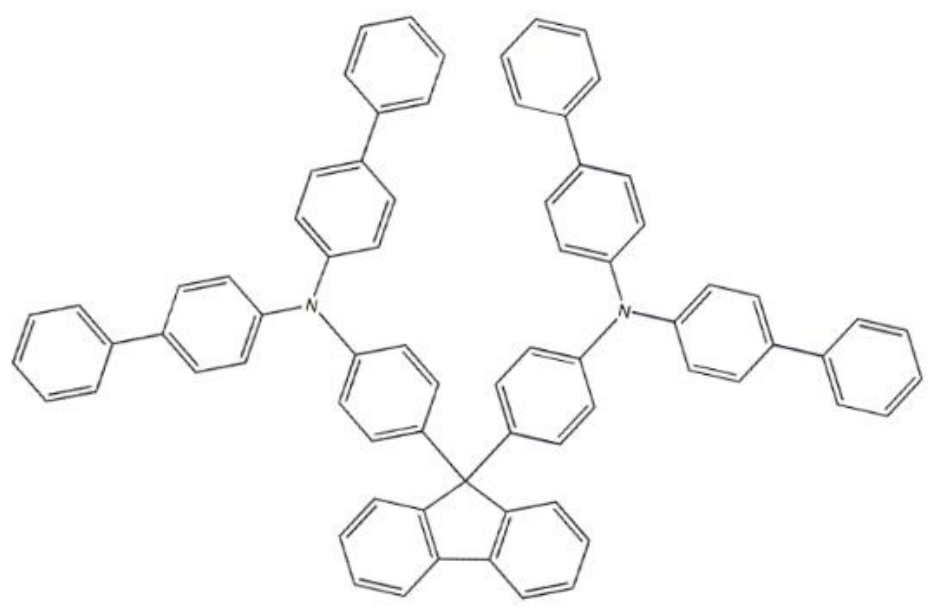

Figure S4: Chemical structure of 9,9-bis[4-(N,N-bis-biphenyl-4-yl-amino)phenyl]-9H-fluorene (BPAPF: $\mathrm{C}_{73} \mathrm{H}_{52} \mathrm{~N}_{2}, \mathrm{CAS}_{-\mathrm{Nr}}$ : 174141-92-5). 


\section{Azimuthal intensity distribution in 2D-GIWAXS measurements}
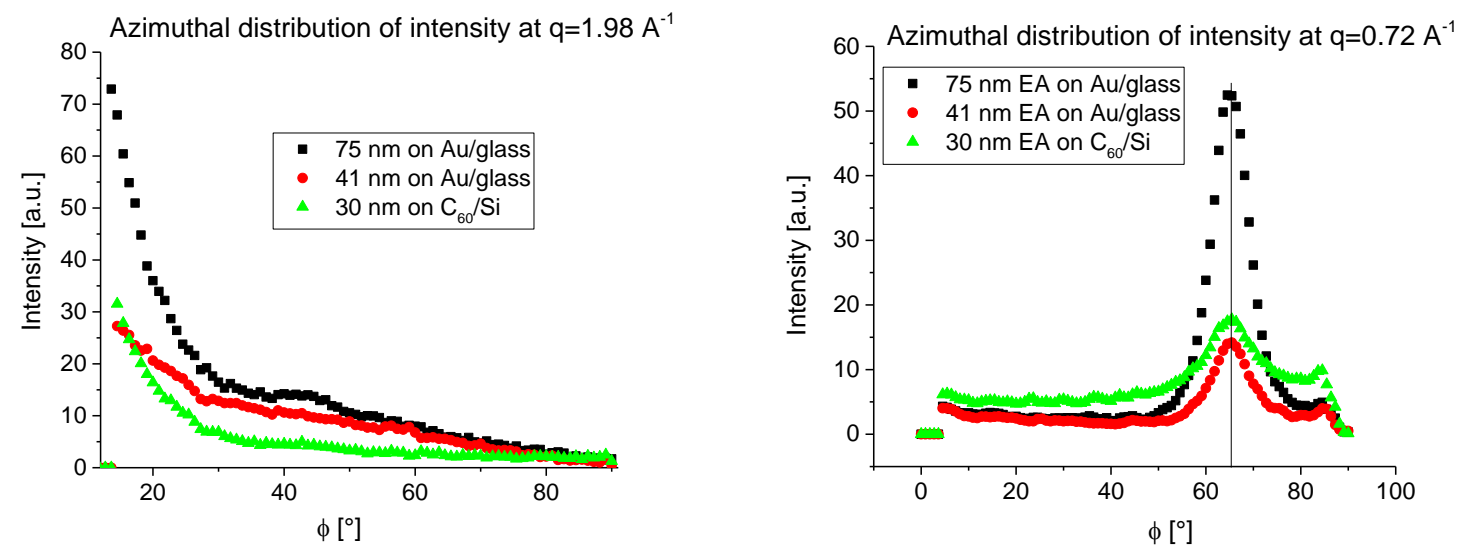

Figure S5: Azimuthal intensity distribution of GIWAXS measurements (Fig. 3 in the manuscript) at the value of the scattering vector $\mathrm{q}=1.98 \AA^{-1}$ (corresponds to (102) $\pi$-stacking lattice planes) (a), and $\mathrm{q}=0.72 \AA^{-1}((011)$ planes) (b) for ellagic acid thin films on $\mathrm{C}_{60} / \mathrm{Si}$ and $\mathrm{Au} / \mathrm{glass}$. For evaluation of the full maximum at half width (FWHM) of the reflection at $\mathrm{q}=1.98 \AA^{-1}$ data was background corrected, and the reflection modeled to a $50 \%$ Gaussian, $50 \%$ Lorentzian function. FWHM of $13^{\circ}\left(75 \mathrm{~nm}\right.$ film), $16^{\circ}$ (41 nm film), and $13^{\circ}\left(30 \mathrm{~nm}\right.$ film on $\mathrm{C}_{60}$ ) were obtained.

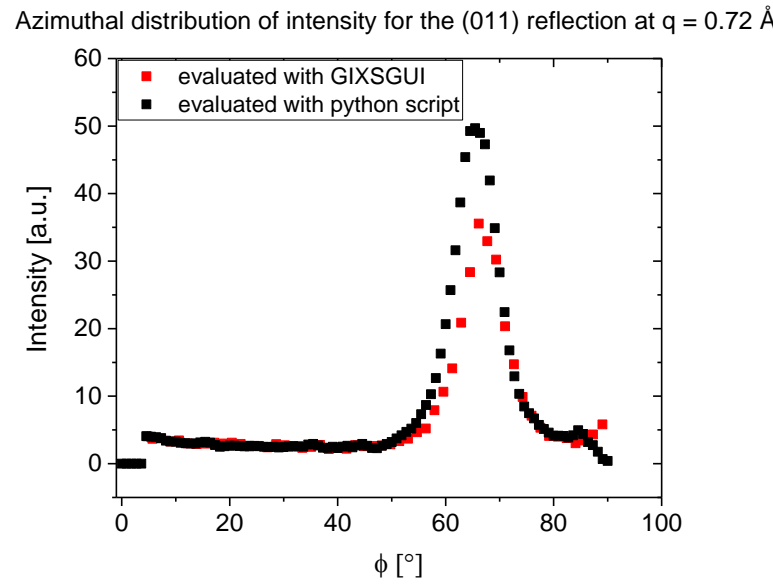

Figure S6: Comparison of the evaluation of radial intensity $I(\phi)$ using the function linecut restrained to $q \in\left[0.66 \AA^{-1}, 0.8 \AA^{-1}\right]$ of the free software GIXSGUI (red squares), and using Gaussian distributed weights of an own python script for $\mathrm{q}=0.72 \AA^{-1}$ (black squares). Both methods agree well within the margin of errors. 


\section{LEED patterns of a closed monolayer of $\mathrm{C}_{60}$ and additional ellagic acid}

(a)

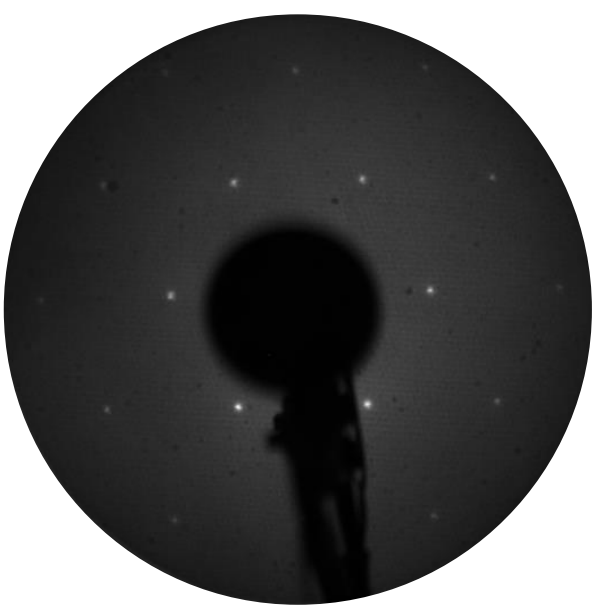

(b)

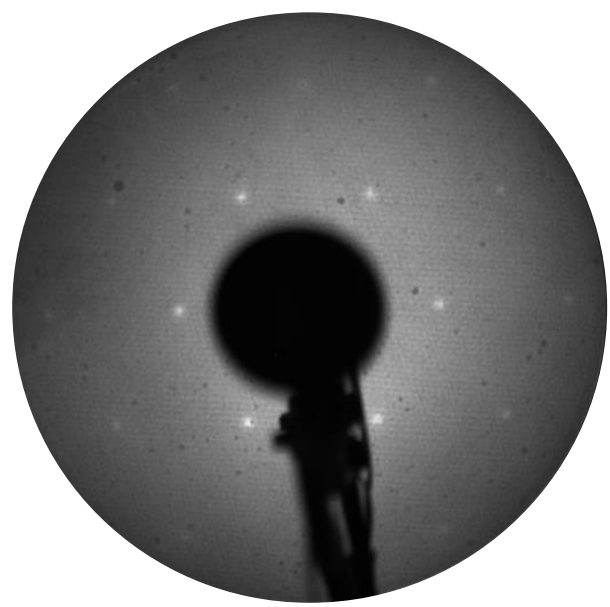

Figure S7: Low-energy electron diffraction pattern of a closed $\mathrm{C}_{60}$ monolayer on $\mathrm{Ag}(111)$. (a) Before the deposition of elegiac acid. (b) After deposition of 5 monolayer equivalents of ellagic acid, resulting in a noticeably diminished contrast. The primary electron energy was $40 \mathrm{eV}$ in both cases. 


\section{Supplementary AFM images and analysis}


a) Height

$400.0 \mathrm{~nm}$

b)
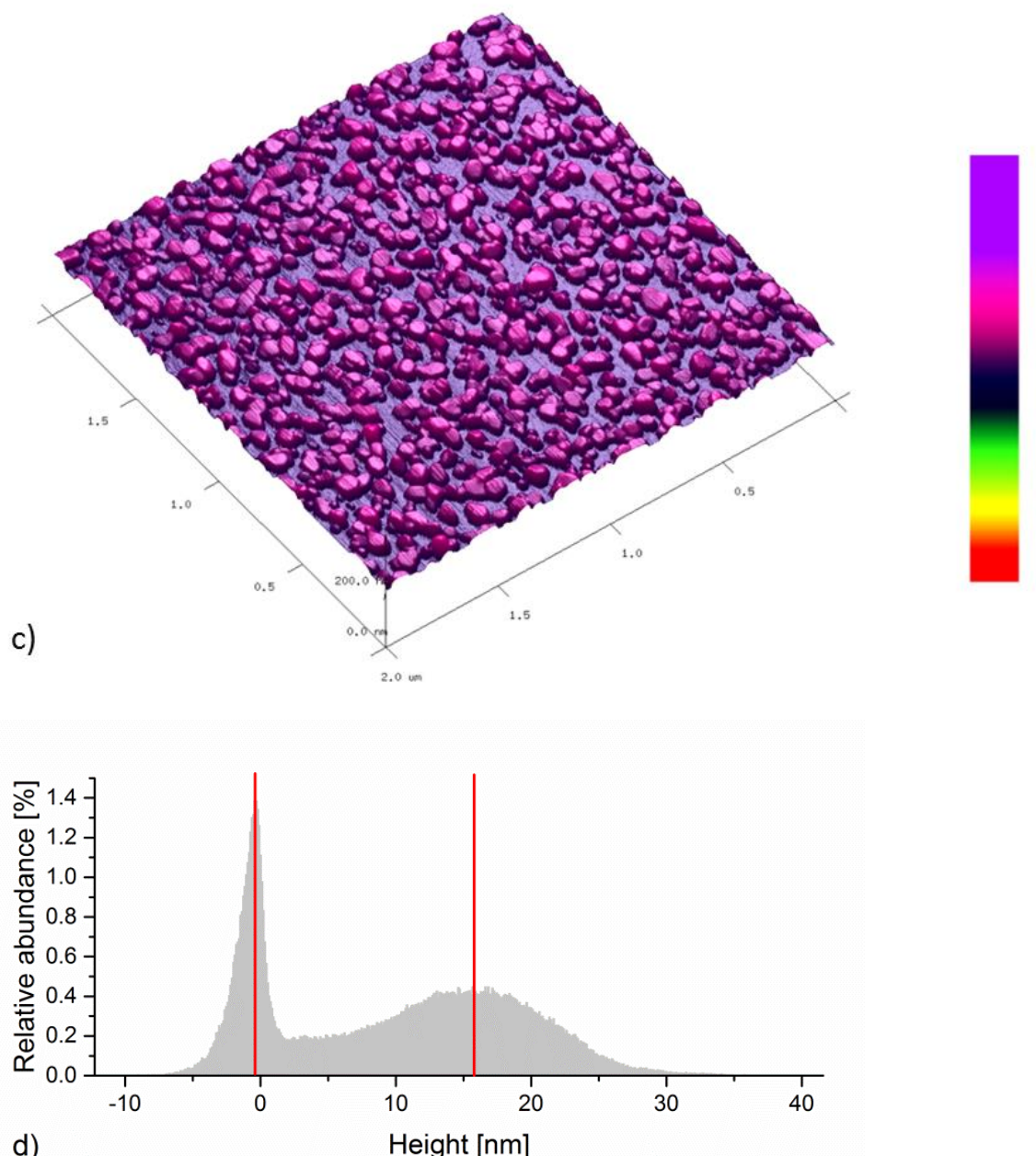

d)

Height $[\mathrm{nm}]$

Figure S8: AFM images for a $30 \mathrm{~nm}$ ellagic acid (EA) film on Floatzone-Si (a), a $41 \mathrm{~nm}$ EA film on Au/glass substrate (b), a 3D reconstruction of the AFM image of $16 \mathrm{~nm}$ EA deposited on Floatzone-Si (c) as shown in the manuscript in Fig. 6, and histogram data of the AFM image after applying the flattening procedure (d). The thickness of the EA film is derived as the histograms relative depth between the maxima for the silicon surface and the EA film. 


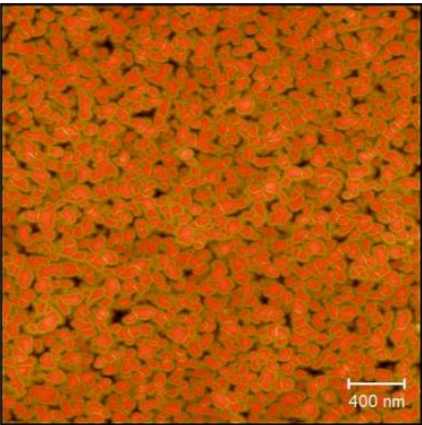

a)

Mask

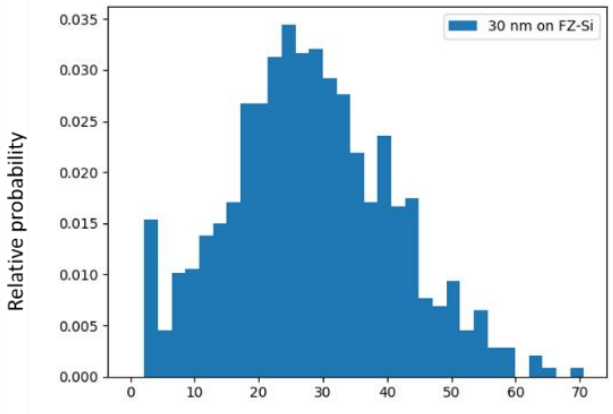

b)

Figure S9: Grain location (Number of steps: 10, drops size: 1\%, threshold: $10 \mathrm{px}^{2}$ ) and segmentation (Number of steps: 50, drop size : 3\%) using the watershed algorithm of the free software Gwyddion for an AFM image of $30 \mathrm{~nm}$ ellagic acid on silicon (a), and the histogram of the equivalent disk radii of the segmentation (b).

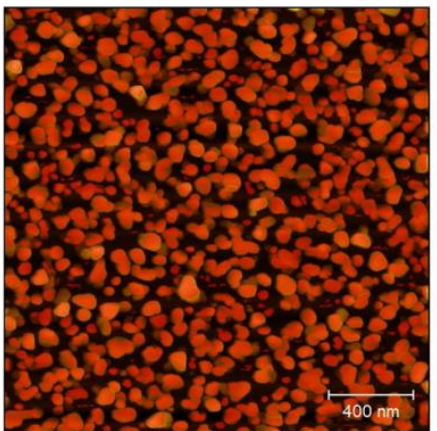

a)

Mask

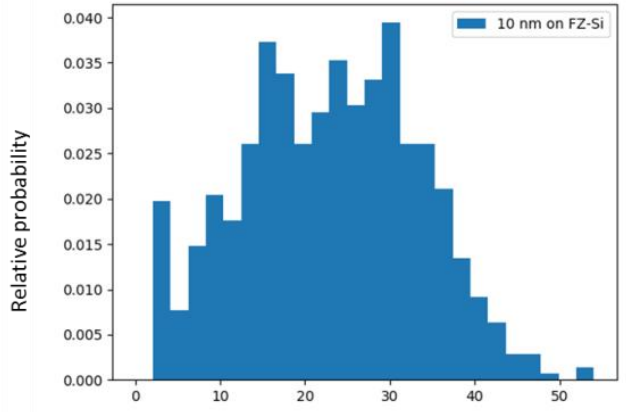

b)

Equivalent disk radius $[\mathrm{nm}]$

Figure S10: Grain location (Number of steps: 10, drops size: 2\%, threshold: $10 \mathrm{px}^{2}$ ) and segmentation (Number of steps: 50, drop size : 4\%) using the watershed algorithm of the free software Gwyddion for an AFM image of $16 \mathrm{~nm}$ ellagic acid on silicon (a), and the histogram of the equivalent disk radii of the segmentation (b). 


\section{UV-VIS spectrum and optical dispersions of ellagic acid thin films}

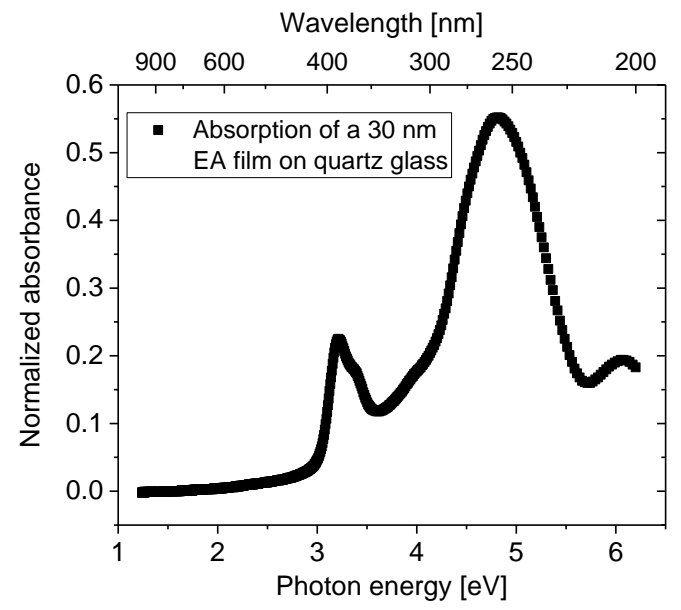

Figure S11: Absorption spectrum of a $30 \mathrm{~nm}$ ellagic acid thin film on quartz glass substrate. The measurement was done at a Shimadzu UV-1800.
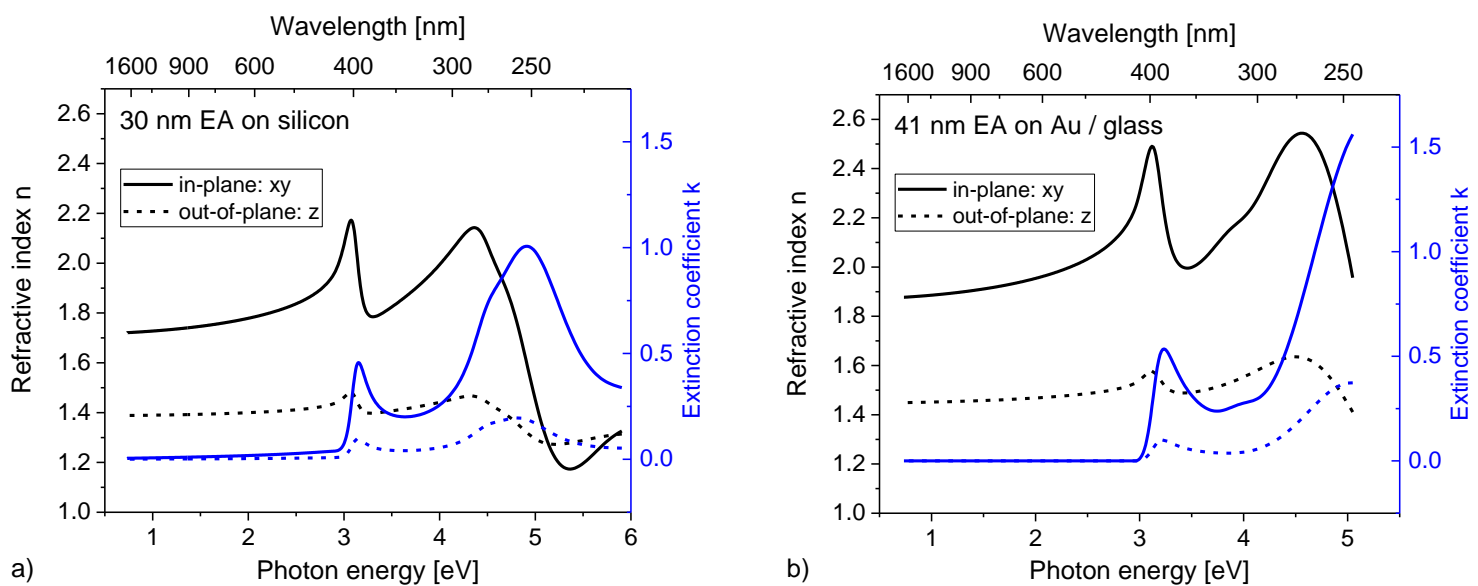

Figure S12: Optical dispersion of ellagic acid (EA) films: $30 \mathrm{~nm}$ (a) on silicon substrate, and $41 \mathrm{~nm}$ on Au/glass (b). 


\section{Optical modeling of substrates and roughness}

Before the deposition of ellagic acid thin films on floatzone (FZ) silicon or Au/glass, the ellipsometric data of the substrates was recorded and modeled as follows: The optical dispersion of Si was taken from the database (CompleteEASE software 6.51, J.A. Woollam Co., Inc). For the Au/glass substrates the opaque $100 \mathrm{~nm}$ thick Au layer was modeled by a B-Spline function based on the optical dispersion of gold from the database.

Optical roughness of the ellagic acid thin films was modeled as a top layer with $50 \%$ void fraction and $50 \%$ ellagic acid fraction (uniaxial optical dispersion) using an effective medium approximation (EMA) according to Bruggeman [Aspnes, D. E. Thin Solid Films 1982, 89 (3), 249] for the nominal $100 \mathrm{~nm}$ and $50 \mathrm{~nm}$ films on gold and the $30 \mathrm{~nm}$ film on silicon. The thicknesses of the optical roughness layers were evaluated as $1.2 \mathrm{~nm}, 1.3 \mathrm{~nm}$, and $0.0 \mathrm{~nm}$, respectively. Therefore, the addition of optical roughness in the ellipsometric modeling did not affect the optical dispersion of ellagic acid significantly. For the thinnest film of nominal $10 \mathrm{~nm}$ ellagic acid on silicon, including an optical roughness layer in the fit leads to a strong correlation of the fit parameters. Thus, the ellipsometric data for the nominal $10 \mathrm{~nm}$ thick film is modeled without optical roughness. Differences between roughness values determined by AFM and ellipsometry are observed occasionally. Relations between roughness estimates from ellipsometry and AFM (root means square height $S_{\mathrm{q}}$ ) are complex, and depend also on the exact surface morphology [Yanguas-Gil, A., \& Wormeester, H. 2013, Chapter 4, In M. Losurdo, \& K. Hingerl (Eds.), Ellipsometry at the Nanoscale. Heidelberg, Berlin, New York: Springer]. 


\section{Supplemental IR-Analysis of ellagic acid thin films on Au/glass and on silicon}



Figure S13: Infrared reflection absorption spectroscopy (IRRAS) spectra of $75 \mathrm{~nm}$ ellagic acid on Au/glass. Due to the metallic surface, thin film interferences can lead to artefacts in the absorption spectrum, as visible between $1800-3000 \mathrm{~cm}^{-1}$.

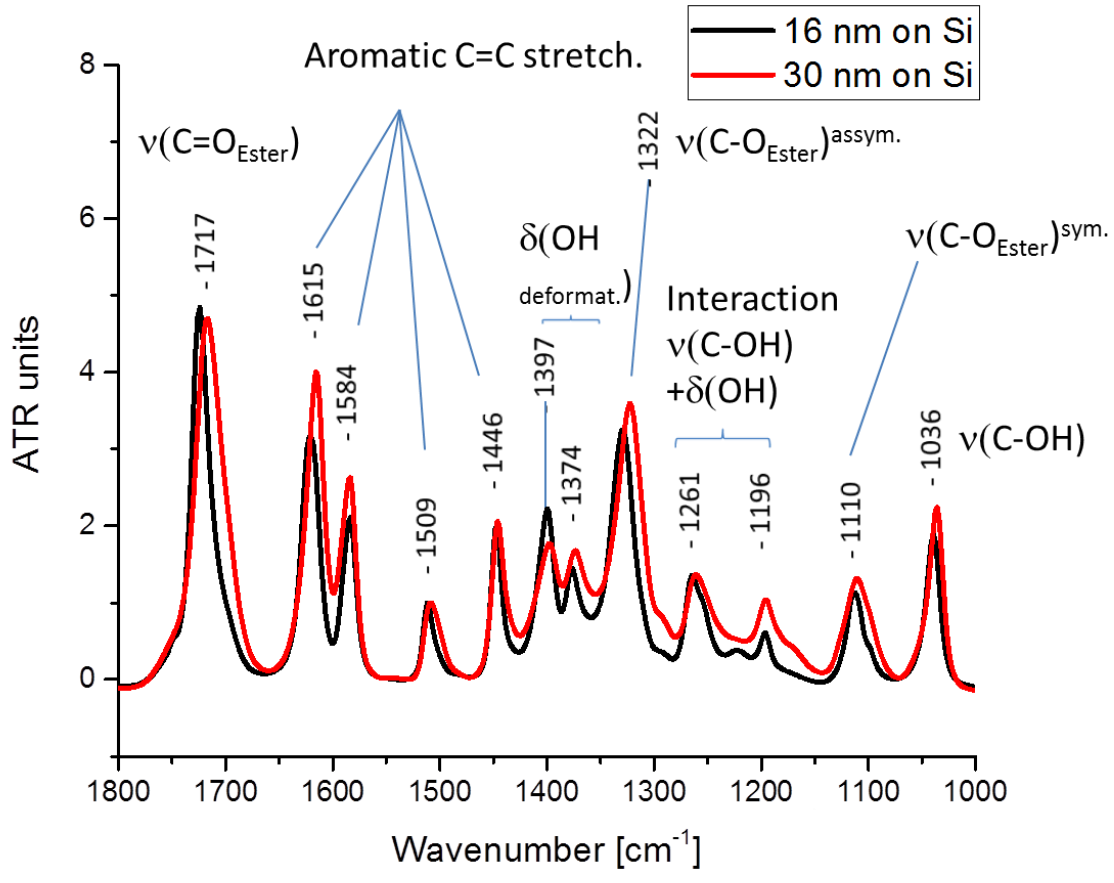

Figure S14: Fingerprint region of absorption of ellagic acid (2,3,7,8-Tetrahydroxychromeno[5,4,3-cde]chromene-5,10-dione) thin films on silicon wafer. The spectra are normalized to $\mathrm{A}_{1510}$. Absorption bands are assigned to stretching vibrations of the lactone group $\left(v\left(\mathrm{C}=\mathrm{O}_{\text {Ester }}\right), v\left(\mathrm{C}-\mathrm{O}_{\mathrm{Ester}}\right)^{\text {assym. }}, v\left(\mathrm{C}-\mathrm{O}_{\text {Ester }}\right)^{\text {sym. }}\right)$, of the aromatic $\mathrm{C}=\mathrm{C}$ bonds, of the $\mathrm{C}-\mathrm{OH}$ bonds $(v(\mathrm{C}-\mathrm{OH}))$, and bending vibrations of the $\mathrm{OH}$ groups $(\delta(\mathrm{OH}))$. A shift in the absorption bands between the $16 \mathrm{~nm}$ and the $30 \mathrm{~nm}$ ellagic acid film is visible, most prominent in the vibrations of $v\left(C=\mathrm{O}_{\text {Ester }}\right)$ and $v\left(\mathrm{C}-\mathrm{O}_{\mathrm{Ester}}\right)^{\text {assym. }}$, which is reflecting the presence of a higher amount of weak $\mathrm{H}$-bonding in the $16 \mathrm{~nm}$ ellagic acid film. 


\section{Microscopic images of micrometer-sized ellagic acid crystals}

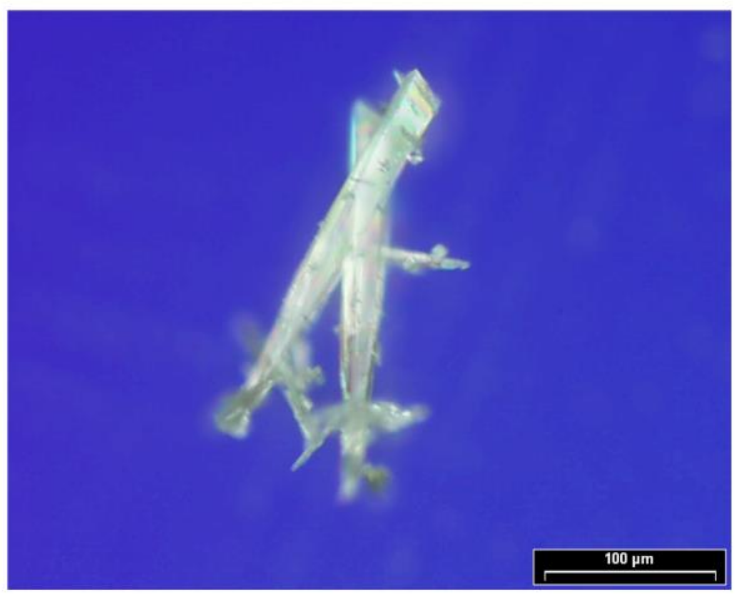

(a)

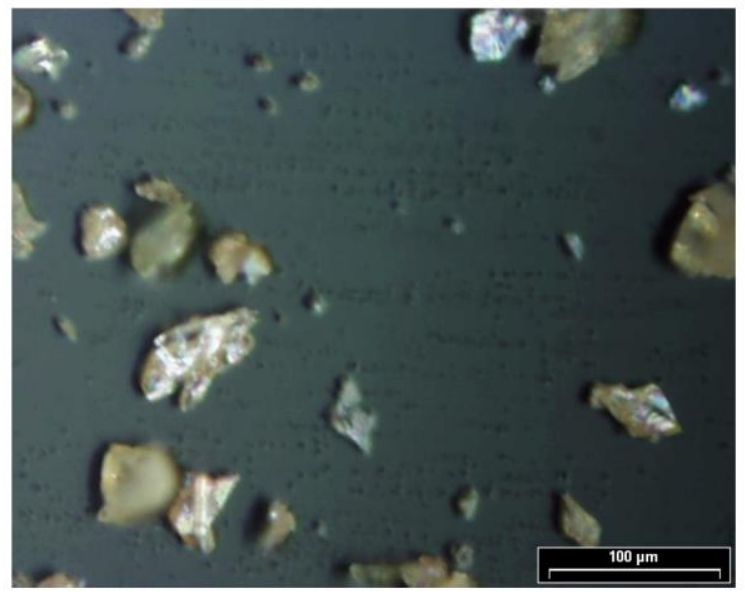

(b)

Figure S15: Microscopic images (Olympus BX40 microscope with Olympus DP10 camera, using linear polarisator and fixed retarder) of the crystals in the ellagic acid powders after sublimation for powder P1 (a) (temperature of deposition: $\vartheta \leq 260{ }^{\circ} \mathrm{C}$, crystalline needles as described in the literature for ellagic acid crystals of the $\mathrm{P} 2{ }_{1} / \mathrm{c}$ crystal system), and powder $\mathrm{P} 2$ (b) $(\vartheta>260$ ${ }^{\circ} \mathrm{C}$ ). P2 was used for the layer preparation and for the LEED analysis, while P1 was used for the IR-analysis. It was proven by solvent absorption spectroscopy (not shown) that both crystalline forms represent indeed the same chemical species. 